\title{
Artificial Heliotropism and Nyctinasty Based on Optomechanical Feedback and No Electronics
}

\author{
Bilge Baytekin,,2 S. Doruk Cezan, H. Tarık Baytekin,2 and Bartosz A. Grzybowski
}

\begin{abstract}
Although plants are typically not considered an inspiration for designing motile robots, they do perform a variety of intricate motion patterns, including diurnal cycles of sun tracking (heliotropism) and leaf opening (nyctinasty). In real plants, these motions are controlled by complex, feedback-based biological mechanisms that, to date, have been mimicked only in computer-controlled artificial systems. This work demonstrates both heliotropism and nyctinasty in a system in which few simple, but strategically positioned thermo-responsive springs and lenses form a feedback loop controlling these motions and substantiating a behavioral analogy to "plants." In particular, this feedback allows the "artificial plant" to reach and stabilize at a metastable position in which the solar flux on the "plants" and the solar power "leaves" are maximized. Unlike many soft robotic systems, our "plants" are completely autonomous, in that, they do not require any external controls or power sources. Bioinspired designs such as this could be of interest for soft robotic systems in which materials alone - rather than power-consuming electronic circuitry—control the motions.
\end{abstract}

Keywords: plant robots, material feedback, artificial heliotropism, artificial nyctinasty

\section{Introduction}

B IOINSPIRED, BIOMIMETIC, AND bionic approaches ${ }^{1}$ are transforming many branches of robotics, endowing the robots' adaptability, agility, and flexibility to interact with their environment. ${ }^{2-4}$ While a rich robotic "fauna" now exists featuring many examples of animal-like robots, the "flora" includes only few examples inspired by sensory behavior/ motion $^{6}$ and osmotic actuation of plants' roots. ${ }^{7-9}$ This scarcity is in sharp contrast to material research (where plants have provided inspiration for multibillion-dollar applications such as Velcro and self-repairing or self-cleaning surfaces) and can stem from the fact that robotics deals with (loco)motion, whereas plants are sessile organisms. Yet, it is known since Darwin $^{10}$ that plants can perform various types of motions toward or away from environmental stimuli (light, chemicals, humidity, gravity, electric field, temperature, or touch), ${ }^{11,12}$ sometimes on very fast time scales (milliseconds to seconds). ${ }^{13}$

In particular, the ability to rapidly open and close the petals upon light exposure (nyctinasty) ${ }^{14}$ and turn the leaves toward the sun (heliotropism) ${ }^{11,12}$ are among the most fascinating, "adaptive" properties of plants. These diurnal motions are underlain by specialized biochemical interactions and, in the case of heliotropism, by a feedback mechanism in which the socalled motor cells control the orientation of the leaves by changing the turgor pressure upon illumination. ${ }^{15}$ Although various types of solar-tracking systems have been designed, ${ }^{16-19}$ the majority could mimic these phenomena only under preprogrammed computer control. Purely "passive trackers"that is, those not using any electronics, programming, or motors-have also been considered based on light-induced expansion/contraction of various materials (e.g., shape memory alloys $^{20}$ ) by light or vapor pressure generation by heat. However, such systems ${ }^{16-19}$ have not been equipped with any feedback mechanisms that would allow them to track the sun continuously and stabilize in a position maximizing the photon flux at a given sun elevation-instead, they could only change between "binary" unbent/fully bent states.

In this study, we demonstrate both nyctinasty and heliotropism (Fig. 1) in an autonomous system that is free of any electronics and instead relies on an in-built optomechanical feedback, to actuate, control, and self-regulate the robotic plant-like motion. Using few appropriately positioned nitinol $^{21,22}$ springs and lenses, our "plants" (called so by behavioral analogy to real plants) open upon light exposure and can track the sun until the feedback mechanism stabilizes the

\footnotetext{
${ }^{1}$ Department of Chemistry, Bilkent University, Ankara, Turkey.

${ }^{2}$ UNAM-Materials Science and Nanotechnology Institute, Bilkent University, Ankara, Turkey.

${ }^{3}$ IBS Center for Soft and Living Matter and the Department of Chemistry, Ulsan National Institute of Science and Technology, UNIST, Ulsan, Korea.
} 
a
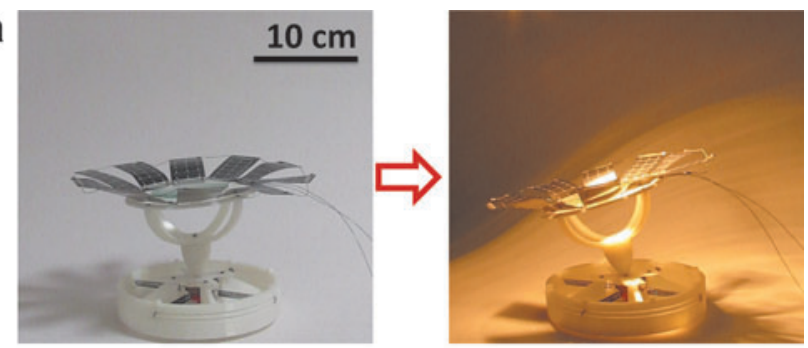

b
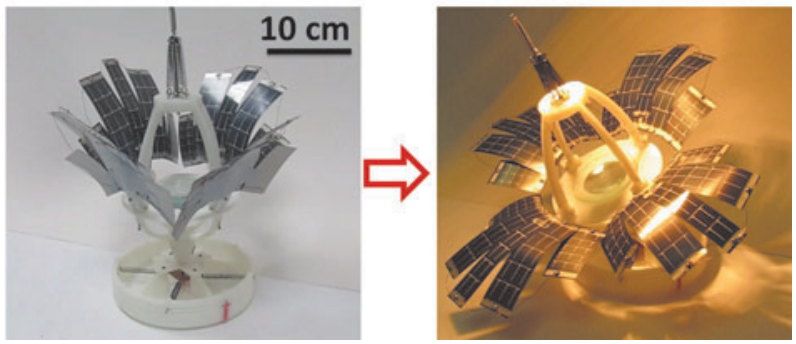

FIG. 1. Systems exhibiting heliotropism and nyctinasty. (a) Two images of a heliotropic system in the dark (left) and bent toward the source of light (right). See also Supplementary Movie S3. (b) Two images of a system exhibiting both heliotropism and nyctinasty. The image on the left is for the "plant" in the dark, with its "leaves" closed. The image on the right shows the same device with the leaves open and the stem bent toward the source of light. See also Supplementary Movie S4. Both assemblies comprise a three-dimensional printed plastic body (for specific parts, see Supplementary Figs. S2 and S5), appropriately positioned nitinol springs, lenses on top of the stem, and solar panels serving as "leaves."

solar-panel "leaves" in a nonequilibrium, dissipative state ${ }^{23,24}$ harnessing the maximal possible amount of sunlight. Field tests show that compared to the nonheliotropic system, our device increases the efficiency of light harvesting by $30-110 \%$ (depending on the elevation of the sun), and can store this collected energy in an internal battery or a capacitor. Its inexpensive components, minimization of energy consumption by eliminating motion-controlling electronics, and complete autonomy and robustness (no easy-to-break parts) make this design concept appealing especially for harsh environments (e.g., deserts and space exploration), in which maintenance of more delicate computer-controlled solar trackers might be problematic. In a wider context, materials with internal feedbacks are interesting as control elements of new classes of adaptive systems and autonomous, continuum-motion robots not requiring external power sources. ${ }^{2,3,25-29}$

\section{Materials and Methods}

\section{Choice of responsive materials}

While feedbacks are essential to the functioning of living matter, ${ }^{30-33}$ engineering them into purely material-based systems - that is, systems without any preprogrammed electronic components-has generally been a challenge, with only few examples of rational design. ${ }^{34,35}$ Searching for suitable feedback mechanisms for our heliotropic systems, various phenomena in which light could potentially induce directional motion have been considered. Pressure-driven systems (in which lightinduced heating of a fluid or gas enclosed in a soft material would cause this material to deform) or light-induced deformations of polymers both exerted forces that were too small to move com- ponents of large "plants." Systems based on electromagnetic induction and feedback could track light in one dimension (Supplementary Fig. S1 and Supplementary Movie S1; Supplementary Data are available online at www.liebertpub.com/soro), but their extension to several degrees of freedom has proven cumbersome. Eventually, we focused on designs incorporating nitinol elements, which have been successfully used in several soft robotics applications. ${ }^{36-42}$ Nitinol is a well-known shapememory alloy composed of $\mathrm{Ni}$ and $\mathrm{Ti}^{21,22}$ with an improved resistance to fatigue, and operating by reversible solid-state phase transformation between two different martensite crystal phases.

\section{Heliotropic plants}

We first discuss the design capable of heliotropism, but not yet nyctinasty. The assembly shown in Figures 1a and 2 comprises a three-dimensional printed body and a stem (cf. Supplementary Fig. S2 for design details) connected to and supported by radially oriented nitinol springs. These springs are made of a $\sim 2 \mathrm{~mm}$ thick nitinol wire, are $\sim 6 \mathrm{~mm}$ in diameter, and have 11 turns per $2 \mathrm{~cm}$ length (total length in the constricted form). Two convex lenses are placed on the stem, one on top of the other (lens diameters 75 and $50 \mathrm{~mm}$, respectively, $\sim 10 \mathrm{~cm}$ focal length for each lens, see Supplementary Fig. S3), and are surrounded by 8-10 solar panels (Flexible Solar Panel MP3-37, MP3-25, or SP3-37 from Powerfilm) serving as "leaves" harvesting the energy of sunlight. Each solar panel can generate 4.5-4.8 V, and when the panels are connected in parallel, they give maximum current of $180-250 \mathrm{~mA}$ depending of the intensity of the impinging light.

The dimensions of the system were adjusted such that the lenses focus the light onto the nitinol springs, causing local heating and contraction. Figure 2 illustrates the response of the system to sun at two different elevations. When the sun is high (left column), that is, when the stem is yet unbent, the initial focal point is near the center of one of the springs, denoted in Figure $2 \mathrm{~b}$ as $\mathrm{x}_{\mathrm{m}}$. As this position is heated and contracted, the stem tilts toward the sun and the focal point gradually "engages" and contracts portions of the spring between $\mathrm{x}_{\mathrm{m}}$ and the innermost region $\mathrm{x}_{\mathrm{i}}$. On the other hand, when the sun is low (right column), the initial focal point is at the spring's outer region, $x_{0}$. The spring is then heated and gradually contracted over its entire extent, from $x_{o}$ to $x_{i}$, causing bending more pronounced than in the case of high sun elevation. Naturally, there is a continuum of cases between the two illustrated extremes and the degree of heliotropic motion changes with the position of the sun; the main point, however, is that the "plant" is always stabilized in the position facing the source of light and can track it continuously (cf. Fig. 3c and Supplementary Movie S2). When the irradiation ceases, the stem returns to its upright position (Fig. 2c and Supplementary Movie S3).

We note that tracking is less efficient when the assembly is initially positioned such that the focused light beam does not trace the springs but, at least at times, falls between these springs; when this happens, the plant slowly "relaxes" to the upright position. This problem is remedied in designs in which more radially directed springs (e.g., eight in Supplementary Movie S5 and also Supplementary Fig. S4) are used and the plant responds to light from a wider range of azimuthal angles. We also observe that because the changes in the temperature of the locally heated springs are small (within $20^{\circ} \mathrm{C}$, see thermal-camera images in Fig. 2b) and the response of NiTi is fast (10-20s), the sun-tracking motions are rapid 


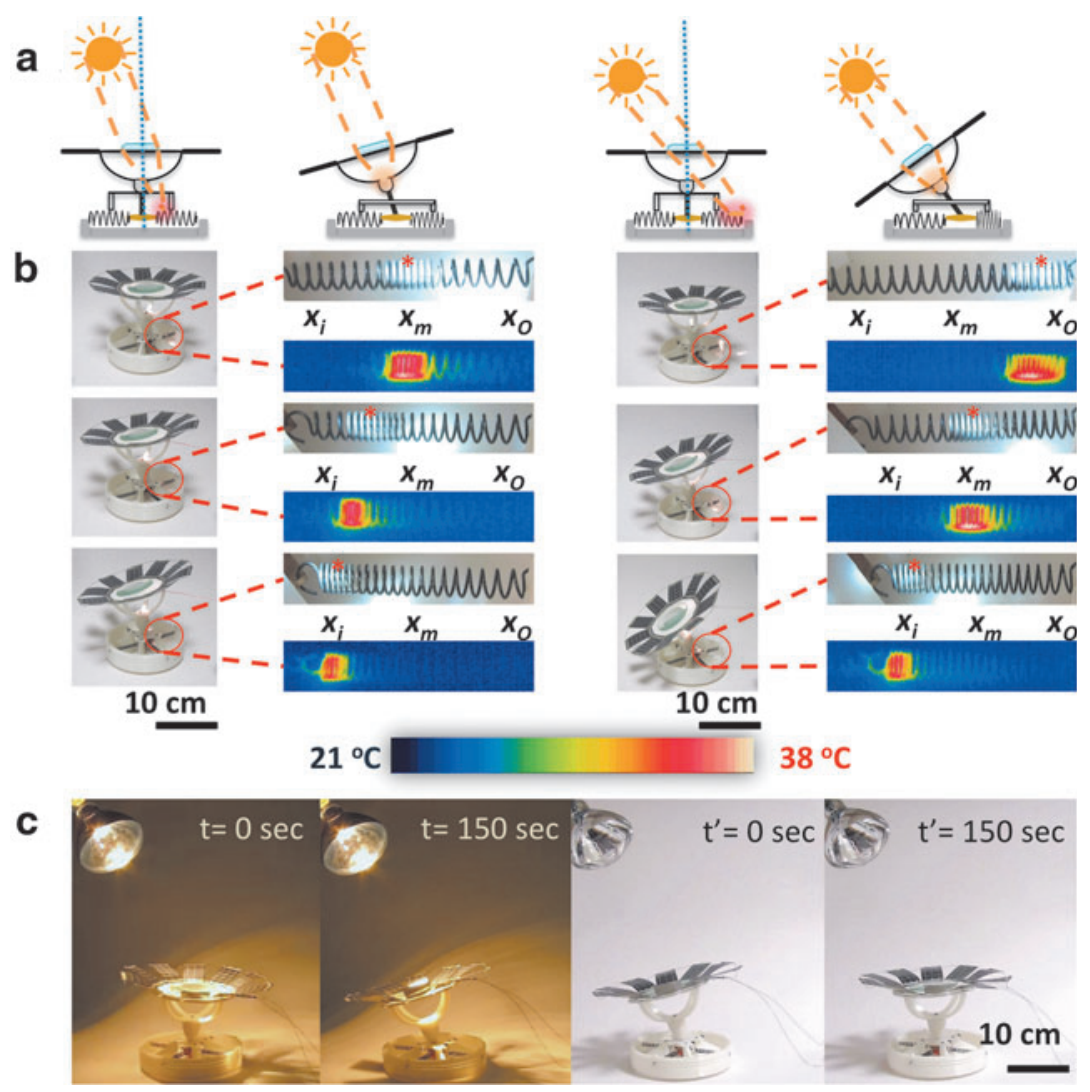

FIG. 2. Schemes and images of heliotropic "plants." The two pairs of schemes in (a) illustrate focusing of light onto nitinol springs whose local heating and contraction cause the stem to bend toward the sun. The left scheme is for the case when the sun is high - then, the spring is gradually being heated from its central region (denoted $x_{m}$ ) toward the innermost region $x_{i}$, resulting in a relatively small contraction and tilting of the stem. In contrast, the scheme in the right column is for the case where the sun is low and the heating starts near the spring's outermost region, $\mathrm{x}_{\mathrm{O}}$. In this case, the spring contracts more and the tilt of the stem is larger. The experimental images for the two scenarios are provided in panel (b). The insets focus on the springs being contracted (focal points are the lighter regions denoted by red asterisks). The heating of the regions onto which light focuses is also illustrated by the thermal camera images (colorful stripes below the optical images of the springs). Gradient scale is for the temperature of the springs and varies from $21^{\circ} \mathrm{C}$ in unheated regions to $38^{\circ} \mathrm{C}$ at the focal point. (c) Illustrates fast response of the system to light exposure (here, to an IR lamp, $250 \mathrm{~W}$, General Electric). The "plant" bends toward the source of light within $2.5 \mathrm{~min}$ and then unbends within 2 min when the light is switched off (see also Supplementary Movie S2).

(compared to those of living plants) and occur on a time scale of 1-2 min (Supplementary Movie S3).

\section{Origin of feedback}

The stabilization at a given position is due to a negative feedback mechanism illustrated in Figure 4. Briefly, as the spring (denoted as " 1 ") is heated and contracted, the stem bends until reaching a steady-state position in which the focal point is near the spring's inner end (cf. Fig. 2). If the spring is overheated or the stem is overturned (e.g., by wind or any other mechanical disturbance), the focal point moves into the space between spring " 1 " and the opposite spring " 2 ," or even onto spring " 2 ." In the first case, the focused beam no longer heats the spring, so it cools down and relaxes; in the latter case, spring " 2 "' is heated and contracted; in both cases, the stem tilts back to its steady-state position, which, as we have seen, depends on the elevation of the sun.

\section{Combining artificial heliotropism with nyctinasty}

Finally, we demonstrated a system exhibiting both heliotropism and nyctinasty. In this design, we augmented the he- liotropic system discussed above with a "crown" of four more nitinol springs placed on top of the stem and connected by threads to the solar panel "leaves" (Figs. 1b and 5, Supplementary Movie S4; see also Supplementary Fig. S5 for details of the crown design and nyctinastic response). Upon irradiation, these springs heated and contracted, and within ca. 23 min, the "plant" opened up its solar panels (Fig. 5b). This nyctinastic movement then exposed the inner lens (same as in the heliotropic design) and the device was able to track the sun as already described. When the light was switched off, the stem returned to the upright position, while the leaves closed up as illustrated in Figure 5c and in Supplementary Movie S4.

\section{Results}

\section{Performance characteristics}

The performance of the "plant" described above was tested in the field (Ankara, Turkey) on both warm $\left(\mathrm{ca} .30^{\circ} \mathrm{C}\right)$ and cold (ca. $10^{\circ} \mathrm{C}$ ) sunny days (Fig. 3). The device followed the sun over a complete diurnal cycle, in the process changing its inclination by ca. $50^{\circ}$ (Fig. 3c). During these motions, the solar panel "leaves" harnessed the energy of sunlight. Importantly, 

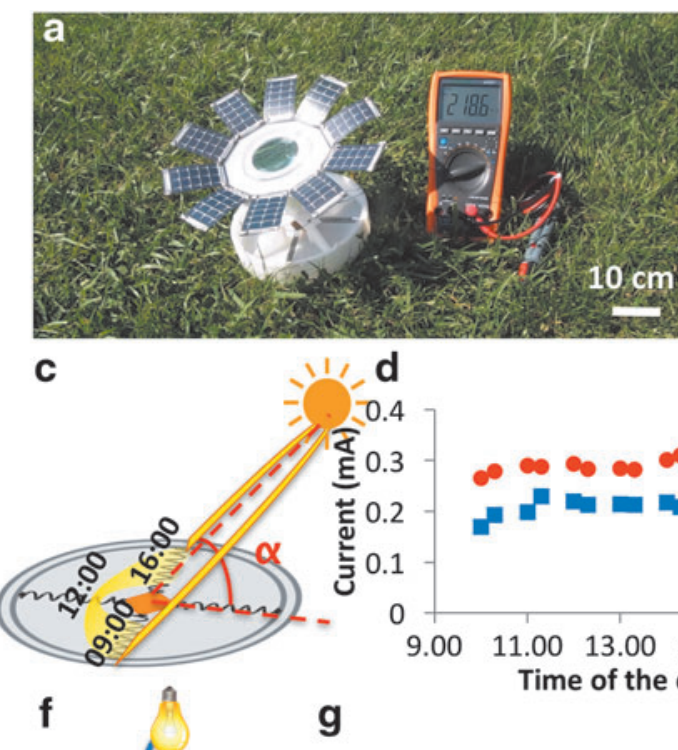

g

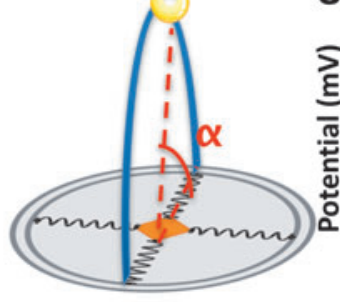

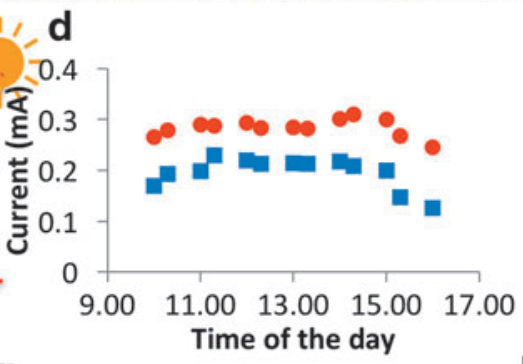

h

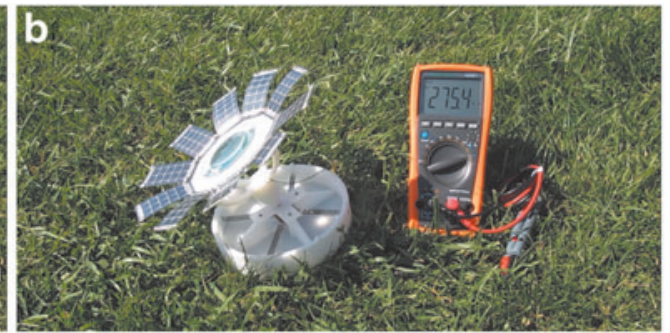

e
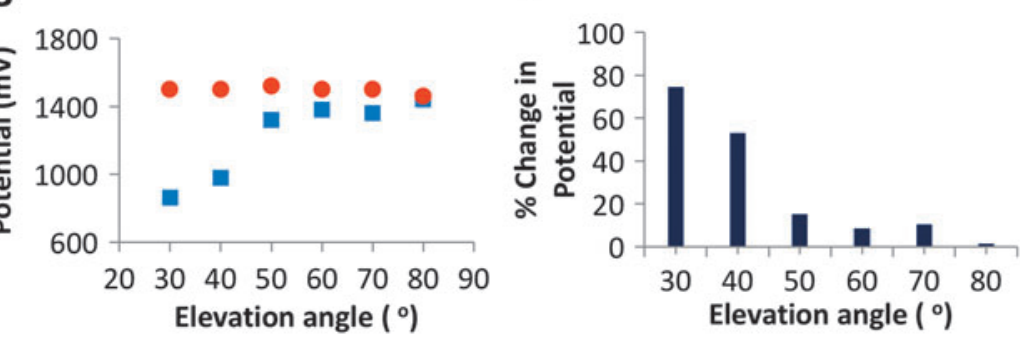

FIG. 3. Field performance of artificial heliotropes on a typical summer day in Ankara, Turkey. (a) Initial position, and (b) final position during heliotropic action at lower sun elevations. (c) Experimental scheme showing the field experiments and the elevation angle of the sun, $\alpha$. (d) Current generated by 10 solar panel "leaves" connected in parallel is plotted as a function of the time of the day (from 9 AM to 5 PM). Red (circle) markers plot the current for the heliotropic device capable of tracking the sun; blue (square) markers are for the device in which the "stem" is not moveable and no sun tracking is possible. Based on the data from (d), panel (e) plots the percentage improvement in power generation for the heliotropic device versus the nonheliotropic, immobile stem control. Power enhancement is up to $110 \%$ when the sun is low and bending of the stem is essential to capture any appreciable flux of photons. (f) Experimental scheme showing the laboratory experiments and the elevation angle of the light source, $\alpha .(\mathbf{g}, \mathbf{h})$ A similar comparison (heliotropic vs. nonheliotropic design), but based on the ability to load a capacitor connected to the solar panels of the "plant" (for the wiring scheme and further details, see Supplementary Fig. S6). (In (g), Red [circle] markers=heliotropic plant, blue [square] markers $=$ nonheliotropic plant.)

FIG. 4. The optomechanical feedback stabilizing the plant in the position maximizing solar flux. When spring denoted as " 1 " is heated by the sun beam focused through the lens, it contracts and the stem turns toward the sun until the steady-state position is reached. Any additional overturning is prohibited since the beam is then focused between the two springs, or onto spring " 2 ," in both cases causing retraction of the stem. These two situations are illustrated by the experimental images in the bottom row. Red asterisks give the position of the focal point.
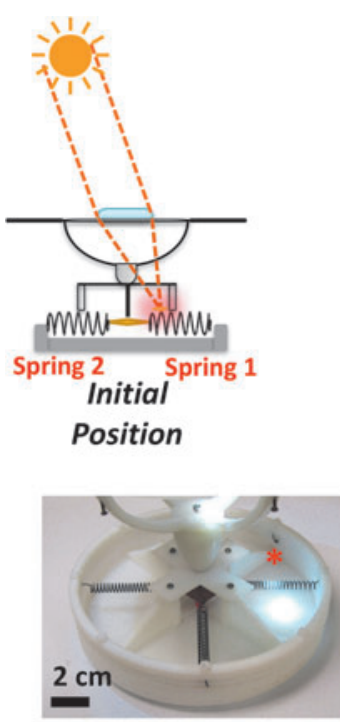

Overturn 1

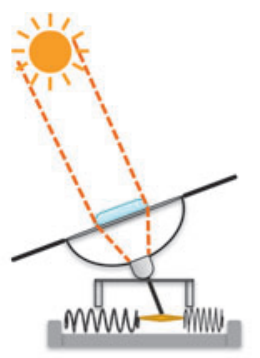

Heliotrope (Steady-state)

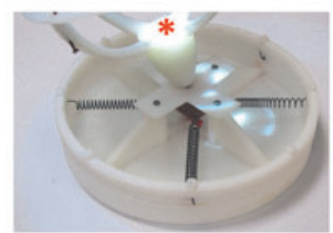

Heliotrope

(Steady-state)

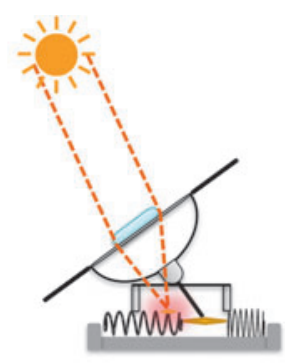

Overturn

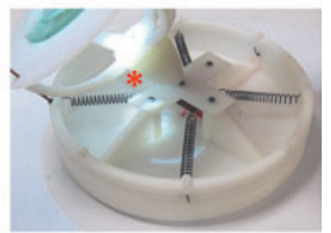

Overturn 2 


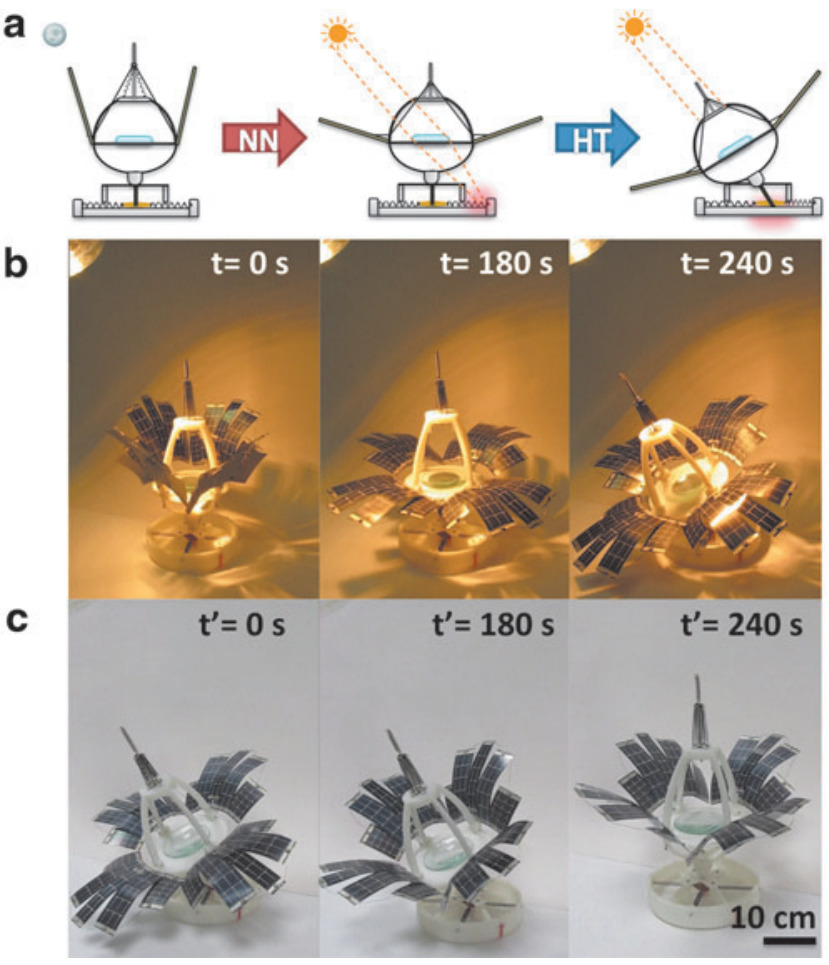

FIG. 5. Device capable of both nyctinasty and heliotropism. (a) Scheme of the "plant" exhibiting nyctinastic (NN) and heliotropic (HT) motions simultaneously. Upon light exposure, four nitinol wires at the top of the "crown" contract such that 16 solar panel leaves open up exposing the inner lens that can then control heliotropic motions. Both nyctinasty and heliotropism cease when the light is turned off and the device returns to the initial state. Panel (b) has the experimental images upon irradiation with IR lamp, $250 \mathrm{~W}$, General Electric; images in (c) illustrate "plant's" response when light is turned off. For further details of the design and the integrated nyctinastic and heliotropic motions, see Supplementary Figs. S2, S5, and Supplementary Movie S4.

heliotropism increased the efficiency (over a design in which the stem was held immobile and upright) by $30-110 \%$ depending on the elevation of the sun. The increase was the largest in the morning and in the evening when the sun was at low elevations and illuminated the nonheliotropic "control" plant only at a small angle. In Figure 4d, the efficiency increase is quantified by the power generated by the solar panels, and in Figure $4 \mathrm{e}$ and $\mathrm{f}$, by the potential reached by a capacitor connected to the panels (for details, Supplementary Fig. S6).

\section{Conclusion}

The heliotropic and nyctinastic designs we demonstrated are exceedingly simple in terms of materials used, yet these rudimentary arrangements of springs and lenses can successfully mimic phenomena that in real plants are underlain by elaborate biochemical processes. This is possible because the lens/spring/ solar-panel system is designed to act as a self-powering feedback loop stabilizing the "plants" in the nonequilibrium steady states maximizing solar flux. In fact, we see the design of material-based feedbacks - as opposed to those controlled by electronic elements - as the most important and novel conceptual aspect of this work, and also a challenge for other designs in soft robots. Currently, many forms of feedback loops are known in molecular sciences (e.g., in biochemical networks, ${ }^{32}$ artificial enzymatic networks ${ }^{33}$ chemical oscillators, ${ }^{43}$ or nanoparticle assemblies ${ }^{34}$ ), but the examples are mostly for chemical/material systems, and not yet for robotics. ${ }^{44}$ The key question is then how to choose and arrange macroscopic responsive materials such that they constitute autonomous feedback elements. If realized with durable parts, such material feedback systems can become alternatives to electronic circuits.

\section{Acknowledgments}

Initial stages of this work (on designs still incorporating electronic components) was supported by the Non-Equilibrium Energy Research Center (NERC) at Northwestern University, which is an Energy Frontier Research Center funded by the U.S. Department of Energy, Office of Science, Office of Basic Energy Sciences under award DE-SC0000989. B.B. gratefully acknowledges the support from BAGEP 2016 (Science Academy Young Scientists Program) award and B.A.G. acknowledges the support from the Institute for Basic Science, Korea, Project Code IBS-R020-D1. B.B., S.D.C., and H.T.B. designed and conducted the experiments, B.A.G. conceived the general idea. B.B., H.T.B., and B.A.G. wrote the article. We thank Mr. Murat Dere and Prof. Mehmet Bayındır (UNAM) for their help in thermal imaging.

\section{Author Disclosure Statement}

No competing financial interests exist.

\section{References}

1. Vogel S. Part 3: Solids and Structures. In: Comparative Biomechanics: Life's Physical World. Princeton, NJ: Princeton University Press, 2003; pp.285-491.

2. Pfeifer R, Lungarella M, Iida F. Self-organization, embodiment, and biologically inspired robotics. Science 2007;318: 1088-1093.

3. Ijspeert AJ. Biorobotics: using robots to emulate and investigate agile locomotion. Science 2014;346:196-203.

4. Kim S, Laschi C, Trimmer B. Soft robotics: a bioinspired evolution in robotics. Trends Biotechnol 2013;31:287-294.

5. Rus D, Tolley M. Design, fabrication and control of soft robots. Nature 2015;521:467-475.

6. Dicker MPM, Rossiter JM, Bond IP, Weaver PM. Biomimetic photo-actuation: sensing, control and actuation in sun-tracking plants. Bioinspir Biomim 2014;9:36015.

7. Mazzolai B, Beccai L, Mattoli V. Plants as model in biomimetics and biorobotics: new perspectives. Front Bioeng Biotechnol 2014;2:2.

8. Sadeghi A, Tonazzini A, Popova L, Mazzolai B. A novel growing device inspired by plant root soil penetration behaviors. PLoS One 2014;9:e90139.

9. Mazzolai B, Mondini A, Corradi P, Laschi C, Mattoli V, Dario P. A miniaturized mechatronic system inspired by plant roots for soil exploration. IEEE ASME Trans Mechatron 2011;16:201.

10. Darwin C, Darwin F. The Power of Movements in Plants. London, United Kingdom: John Murray, 1880.

11. Ehleringer J, Forseth I. Solar tracking by plants. Science 1980;210:1094-1098.

12. Hart JW. Plant Tropisms and other Growth Movements. London, United Kingdom: Chapman \& Hall, 1990.

13. Martone PT, Boller M, Burgert I, Dumais J, Edwards J, Mach K, Rowe N, Rueggeberg M, Seidel R, Speck T. 
Mechanics without muscle: biomechanical inspiration from the plant world. Integr Comp Biol 2010;50:888-907.

14. Ueda M, Nakamura Y. Chemical basis of plant leaf movement. Plant Cell Physiol 2007;48:900-907.

15. Häder DP, Lebert M. Photomovement. Amsterdam, Netherland: Elsevier, 2001.

16. Mousazadeh H, Keyhani A, Javadi A, Mobli H, Abrinia K, Sharifi A. A review of principle and sun-tracking methods for maximizing solar systems output. Renew Sustain Energy Rev 2009;13:1800-1818.

17. Luque-Heredia I, Queméré G, Cervantes R, Laurent O, Chiappori E, Chong JY. The Sun tracker in concentrator photovoltaics. In: Next Generation of Photovoltaics: New Concepts (Springer Series in Optical Sciences) Cristobal A, Vega, AM, López AL. (Eds). Berlin, Heidelberg: Springer, 2012, pp. 61-94.

18. Li C, Liu Y, Huang X, Jiang H. Direct Sun-driven artificial heliotropism for solar energy harvesting based on a photothermomechanical liquid-crystal elastomer nanocomposite. Adv Funct Mater 2012;22:5166-5174.

19. Wang E, Desai MS, Lee SW. Light-controlled graphene-elastin composite hydrogel actuators. Nano Lett 2013;13:2826-2830.

20. Poulek V. New low cost solar tracker. Sol Energ Mat Sol Cells 1994;33:287-291.

21. Buehler WJ, Gilfrich JW, Wiley RC. Effect of low-temperature phase changes on the mechanical properties of alloys near composition TiNi. J Appl Phys 1963;34:1475-1476.

22. Wang FE, Buehler WJ, Pickart SJ. Crystal structure and a unique "martensitic" transition of TiNi. J Appl Phys 1965; 36:3232-3239.

23. Whitesides GM, Grzybowski BA. Self-assembly at all scales. Science 2002;295:2418-2421.

24. Warren SC, Guney-Altay O, Grzybowski BA. Responsive and nonequilibrium nanomaterials. J Phys Chem Lett 2012;3: 2103-2111.

25. Wehner M, Truby RL, Fitzgerald DJ, Mosadegh B, Whitesides GM, Lewis JA, Wood RJ. An integrated design and fabrication strategy for entirely soft, autonomous robots. Nature 2016;536:451-455.

26. Philamore H, Ieropoulos I, Stinchcombe A, Rossiter J. Toward energetically autonomous foraging soft robots. Soft Robot 2016;3:186-197.

27. Melhuish C, Kubo M. Collective energy distribution: Maintaining the energy balance in distributed autonomous robots using trophallaxis. In: Distributed Autonomous Robotic Systems 6, Alami R, Chatila R, Asama H. (Eds). Tokyo, Japan: Springer, 2007, pp. 275-284.

28. Tolley M, Shepherd RF, Mosadegh B, Galloway KC, Wehner M, Karpelson M, Wood RJ, Whitesides GM. A resilient, untethered soft robot. Soft Robot 2014;1:213-223.

29. Marchese AD, Onal CD, Rus D. Autonomous soft robotic fish capable of escape maneuvers using fluidic elastomer actuators. Soft Robot 2014;1:75-87.

30. Tsang J, Zhu J, van Oudenaarden A. MicroRNA-mediated feedback and feedforward loops are recurrent network motifs in mammals. Mol Cell 2007;26:753-767.

31. Yi TM, Huang Y, Simon MI, Doyle J. Robust perfect adaptation in bacterial chemotaxis through integral feedback control. Proc Natl Acad Sci U S A 2000;97:4649-4653.

32. Giuseppone N. Toward self-constructing materials: a systems chemistry approach. Acc Chem Res 2012;45:2178-2188.

33. Semenov SN, Wong AS, van der Made RM, Postma SG, Groen J, van Roekel HW, de Greef TF, Huck WT. Rational design of functional and tunable oscillating enzymatic networks. Nat Chem 2015;7:160-165.
34. Lagzi I, Kowalczyk B, Wang DW, Grzybowski BA. Nanoparticle oscillations and fronts. Angew Chem Int Ed 2010;49:8616-8619.

35. He XM, Aizenberg M, Kuksenok O, Zarzar LD, Shastri A, Balazs AC, Aizenberg J. Synthetic homeostatic materials with chemo-mechano-chemical self-regulation. Nature 2012;487: 214-218.

36. Kim S, Hawkes E, Cho K, Jolda M, Foley J, Wood R. Micro artificial muscle fiber using NiTi spring for soft robotics. In: Proceedings of 2009 IEEE/RSJ International Conference on Intelligent Robots and Systems. 2009, pp. 2228-2234.

37. Kim B, Lee MG, Lee YP, Kim Y, Lee G. An earthwormlike micro robot using shape memory alloy actuator. Sensor Actuat A Phys 2006;125:429-437.

38. Trimmer BA, Rogers CB. Caterpillar locomotion: a new model for soft-bodied climbing and burrowing robots. In: Proceedings of the 7th International Symposium on Technology and the Mine Problem. Monterey, CA. DOI: 10.1098/rsif.2006.0163.

39. Sugiyama Y, Hirai S. Crawling and jumping by a deformable robot. In: Experimental Robotics IX (Springer Tracts in Advanced Robotics, vol. 21) Ang MH, Khatib O. (Eds). Berlin, Heidelberg: Springer, 2006, pp. 281-290.

40. Menciassi A, Gorini S, Pernorio G, Dario P. A SMA actuated artificial earthworm. Proc IEEE Int Conf Robot Autom 2004;4,3282-3287.

41. Cianchetti M, Arienti A, Follador M, Mazzolai B, Dario P, Laschia C. Design concept and validation of a robotic arm inspired by the octopus. Mater Sci Eng C 2011;31:1230-1239.

42. Rodrigue H, Wang W, Han MW, Kim TJY, Ahn SH. An overview of shape-memory alloy-coupled actuators and robots. Soft Robot 2017;4:3-15.

43. Epstein I, Pojman J. An Introduction to Nonlinear Chemical Dynamics: Oscillations, Waves, Patterns, and Chaos. New York, NY: Oxford University Press, 1998.

44. Grinthal A, Aizenberg J. Adaptive all the way down: Building responsive materials from hierarchies of chemomechanical feedback. Chem Soc Rev 2013;42:7072-7085.

Address correspondence to: Bilge Baytekin

Department of Chemistry Bilkent University

Ankara 06800

Turkey

E-mail: b-baytekin@fen.bilkent.edu.tr

H. Tarik Baytekin

UNAM-Materials Science and Nanotechnology Institute

Bilkent University

Ankara 06800

Turkey

E-mail: baytekin@unam.bilkent.edu.tr

Bartosz A. Grzybowski

IBS Center for Soft and Living Matter and the Department of Chemistry

Ulsan National Institute of Science and Technology

UNIST-gil 50

Ulju-gun

Ulsan 689-798

Korea

E-mail: nanogrzybowski@gmail.com 